\title{
The role of blood flow in determining the sites of atherosclerotic plaques
}

\author{
Christina M. Warboys, Narges Amini, Amalia de Luca and Paul C. Evans*
}

Address: BHF Cardiovascular Sciences Unit, National Heart and Lung Institute, Imperial College London, 2nd Floor, B-Block Hammersmith Campus, Du Cane Road, London W12 0NN, UK

* Corresponding author: Paul C. Evans (paul.evans@imperial.ac.uk)

Fl000 Medicine Reports 2011, 3:5 (doi:10.3410/M3-5)

This is an open-access article distributed under the terms of the Creative Commons Attribution-Non Commercial License (http://creativecommons.org/licenses/by-nc/3.0/legalcode), which permits unrestricted use, distribution, and reproduction in any medium, provided the original work is properly cited. You may not use this work for commercial purposes.

The electronic version of this article is the complete one and can be found at: http://fl 000.com/reports/m/3/5

\begin{abstract}
Atherosclerosis is a chronic inflammatory disease characterized by the accumulation of lipids and inflammatory cells along the inner walls of arteries, and is an underlying cause of cardiovascular disease. Atherosclerotic lesions develop predominantly at branches, bends, and bifurcations in the arterial tree because these sites are exposed to low or disturbed blood flow, which exerts low/ oscillatory shear stress on the vessel wall. This mechanical environment alters endothelial cell physiology by enhancing inflammatory activation. In contrast, regions of the arterial tree that are exposed to uniform, unidirectional blood flow and experience high shear stress are protected from inflammation and lesion development. Shear stress is sensed by the endothelium via mechanoreceptors and is subsequently transduced into biochemical signals resulting in modulation of proinflammatory signaling pathways. In this article, we address the molecular mechanisms behind the spatial localization of vascular inflammation and atherosclerosis, with particular focus on studies by our own group of two key proinflammatory signaling pathways, the mitogen-activated protein kinase pathway and the nuclear factor-kappa-B pathway.
\end{abstract}

\section{Introduction and context}

Cardiovascular disease accounts for the greatest number of deaths worldwide and currently presents a major burden to health services and society. This burden is set to increase as obesity and diabetes, two major risk factors for the disease, reach epidemic levels. Most resources are currently directed towards surgical intervention and management of advanced cardiovascular disease, but clearly prevention would be preferable. Encouraging lifestyle changes is expected to play a major role in slowing disease progression; however, greater understanding of the disease at the molecular level is of critical importance in informing future treatment strategies and aiding disease prevention.

The build up of atherosclerotic plaques within the arterial tree is the underlying cause of most forms of cardiovascular disease, including coronary artery disease and stroke.
Atherosclerosis is a lipid-driven chronic inflammatory disease in which inflammatory cells, lipids, and extracellular matrix (ECM) accumulate within the artery wall to form plaques which reduce, and may eventually shut down, blood flow through the artery, with devastating consequences for the tissue or organ it supplies. A critical step in lesion formation is the oxidative modification of circulating low-density lipoprotein (LDL) by reactive oxygen species. Oxidized LDL promotes the recruitment of leukocytes from the bloodstream to the arterial wall by triggering the expression of adhesion proteins, chemokines, and other proinflammatory molecules on endothelial cells (such as vascular cell adhesion molecule-1 [VCAM-1] and E-selectin, illustrated in Figure 1). ECM remodeling and alterations in ECM composition during atherogenesis can stimulate proliferation and migration of smooth muscle cells and promote proinflammatory signaling, contributing to the development of the 
Figure I. Leukocyte adhesion and transmigration

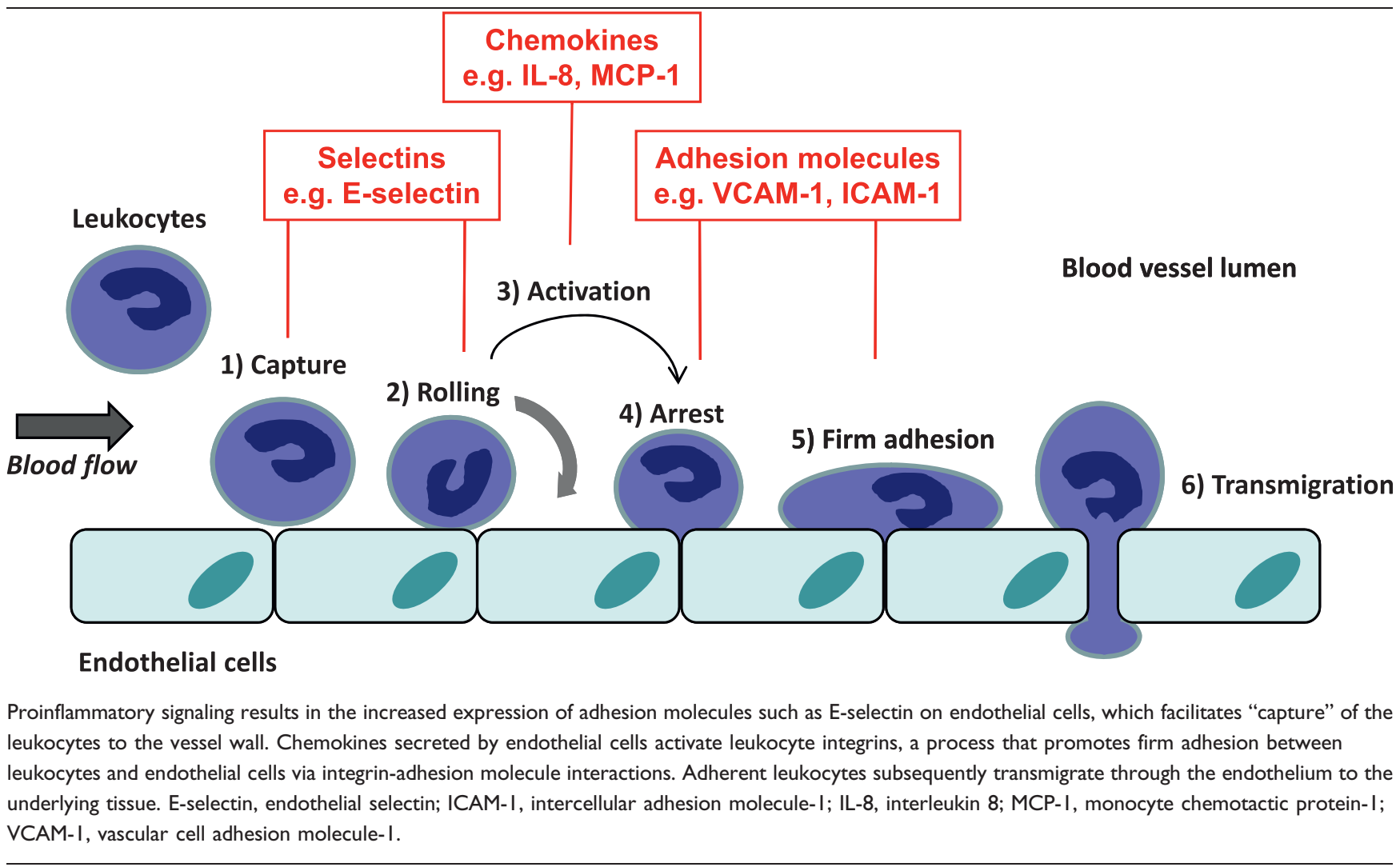

plaque [1]. There is also evidence that endothelial cell apoptosis plays a role in plaque formation, potentially by increasing the permeability of the arterial wall to LDL [2].

Although a wide range of global risk factors such as age, high cholesterol, and obesity significantly increase the risk of developing atherosclerosis, plaque formation occurs predominately at specific sites within the arterial tree, suggesting a critical role for local factors within the vasculature. Atherosclerotic plaques are mainly found at arterial bifurcations and branch points and areas of vessel curvature whereas straight unbranching segments of artery are generally spared. Several molecular mechanisms that influence the localization of plaque formation have been described recently and will be explored within this article.

It was recognized by Caro et al. [3] in 1969 that sites of lesion development were associated with regions of altered blood flow, leading to the suggestion that flowregulated mechanical forces could play a key role in lesion development. Two hypotheses emerged to account for this effect: the mass transport theory and the shear stress theory of atherosclerosis (depicted in Figure 2).
The mass transport theory of atherosclerosis

One possible explanation for the accumulation of atheromatous material at sites of low or disturbed blood flow is that an increased rate of uptake of bioactive substances (e.g., LDL, oxygen, nitric oxide) from the blood stream into the vessel wall results from the increased length of time the blood is in contact with the vessel wall in such areas, particularly in areas of flow stagnation. This idea is supported by a wealth of experiments in animals that have demonstrated greater uptake of labeled macromolecules such as albumin in regions of low blood flow that are susceptible to the development of atherosclerosis [4]. Low or disturbed blood flow might also enhance atherogenesis due to altered transport of leukocytes from the bloodstream to the endothelial surface.

\section{The shear stress theory of atherosclerosis}

The endothelium is in direct contact with flowing blood and hence it is constantly exposed to the mechanical forces that the blood exerts. The drag force exerted by flow on the vessel wall, called shear stress, is a critical factor in maintaining endothelial function and varies with time, magnitude, and direction, according to vascular pulsatility and anatomy. Relatively straight, unbranched arteries are exposed to uniform, unidirectional flow and 
Figure 2. Atherosclerotic plaques occur at sites of low/disturbed blood flow

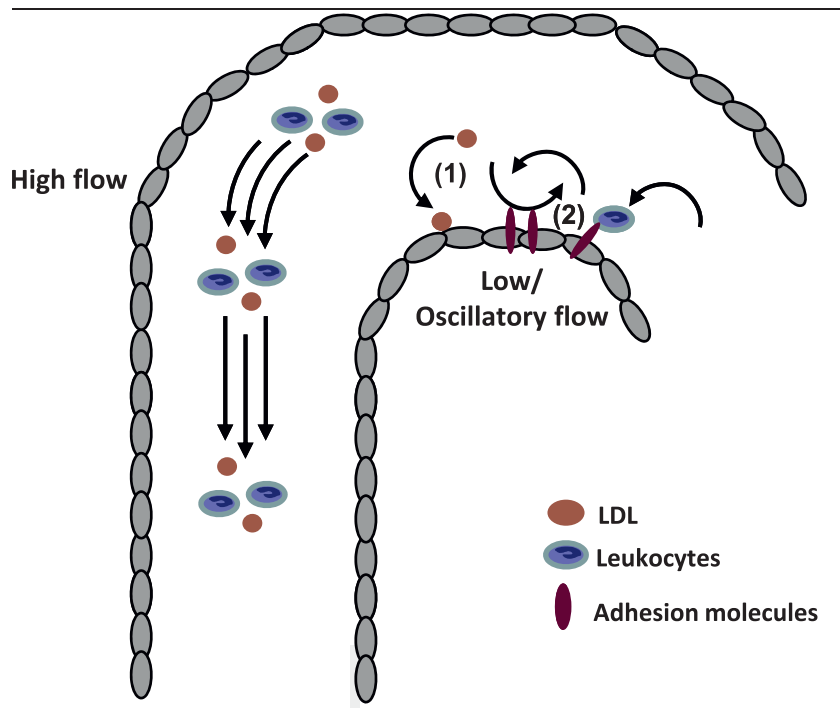

Atherosclerotic lesions form predominantly at regions of the arterial tree exposed to low/disturbed blood flow such as branches, bends, and bifurcations, whilst regions exposed to unidirectional high flow are spared. Two theories have been proposed that account for this localization: (I) the mass transport theory, where atherogenic material such as low-density lipoprotein (LDL) and leukocytes have better access to the arterial wall in areas of low flow or stagnation; and (2) the shear stress theory, where shear stress (mechanical drag) is sensed by endothelial cells, resulting in an altered phenotype. Low/oscillatory shear stress primes endothelial cells for inflammation by inducing adhesion molecule expression.

experience relatively high shear stress (time-averaged mean value approximately $150 \mu \mathrm{N} / \mathrm{cm}^{2}$; equivalent to 15 dynes $/ \mathrm{cm}^{2}$ ). By contrast, at vessel bifurcations and bends, the flow is disturbed and changes direction during the cardiac cycle resulting in relatively low and/ or oscillatory shear stress (time-averaged mean $<50 \mu \mathrm{N} /$ $\mathrm{cm}^{2}$; equivalent to $<5$ dynes $/ \mathrm{cm}^{2}$ ) [5].

Atherosclerosis preferentially develops in regions of flow disturbance and separation, where shear stress is significantly lower in magnitude and exhibits directional variations [3]. Thus low shear stress provides predisposition for atherogenic transformation by contributing to endothelial dysfunction whereas high shear areas might shield against atherosclerosis by enhancing endothelial protection.

For shear stress to influence endothelial cell function, the cell must be able to sense, and then convert, mechanical forces into biochemical signals that alter physiology. A mechanosensory complex on the endothelial cell surface has been identified as an initial transducer of mechanical forces consisting of the adhesion molecules PECAM-1 (platelet endothelial cell adhesion molecule-1), which is the mechanosensor, and VE-cadherin and VEGFR-2 (vascular endothelial growth factor receptor-2), which, once activated, stimulate a host of downstream signaling pathways. Mechanotransduction via this trimolecular complex mediates integrin activation, which causes elongation and alignment of cells in the direction of flow, characteristic of cells in high-shear, protected regions [6]. This is an adaptive response that redistributes and reduces the local mechanical load experienced by the cell, reducing subsequent injury, and is dependent on anchoring of endothelial cells to the ECM via integrins. High laminar shear stress induces rapid conformational activation of integrins leading to remodeling of endothelial attachment sites and increased binding to the ECM, triggering cytoskeletal rearrangement and cell alignment [7].

High shear stress is anti-inflammatory, antiproliferative, antiapoptotic, antithrombotic, and also protective against intracellular endoplasmic reticulum stress [8], which collectively may confer some degree of protection from lesion formation. Here, we focus on the molecular mechanisms that underlie the modulation of inflammatory activation by shear stress.

\section{Shear stress regulates endothelial activation}

Studies of cultured endothelial cells have revealed that prolonged unidirectional high shear stress suppresses proinflammatory activation and leukocyte recruitment in response to an inflammatory stimulus, for example, tumor necrosis factor-alpha (TNF- $\alpha$ ) $[9,10]$. This observation is supported by in vivo studies where endothelial cells at low shear, atherosusceptible sites express proinflammatory markers. In contrast, proinflammatory signaling is suppressed in regions of the arterial tree that are protected from atherosclerosis and exposed to high shear stress [11-14].

Understanding the mechanisms by which high shear stress reduces proinflammatory signaling is an area of active research. In vitro and in vivo studies have revealed a plethora of genes that are altered by shear stress, albeit with a wide disparity between studies, which can be attributed to the different cell types studied and variation in magnitude, direction, and duration of shear stress. Nevertheless, two key proinflammatory signaling pathways: the mitogen-activated protein kinase (MAPK) pathway and nuclear factorkappa-B (NF- $\mathrm{B}$ ) pathway have been identified as being differentially regulated at protected and susceptible sites of the arterial tree $[12,15,16]$. It is likely that both contribute to inflammation and lesion development at susceptible sites since several proinflammatory genes 
implicated in atherosclerosis (e.g., the genes encoding VCAM-1, E-selectin, and IL-8) require the activation of both pathways for transcription to occur. High laminar shear stress suppresses inflammation in part by modulating or inhibiting NF- $\mathrm{BB}$ and MAPK signaling, and the underlying mechanisms are discussed in this article.

\section{The MAPK Pathway}

The MAPKs are a group of highly conserved serine/ threonine protein kinases that play an important role in many cellular processes including apoptosis, proliferation, and inflammation. The three well characterized MAPK pathways are the c-Jun N-terminal kinase (JNK), the p38, and the extracellular signal-regulated kinase (ERK) pathways. The JNK and p38 pathways are preferentially activated by inflammatory cytokines and stress, whereas the ERK pathway is preferentially activated by growth factors. Typically, activation of MAPK pathways is initiated by proinflammatory mediators via activation of a cell surface receptor, activating a chronological phosphorylation cascade. Activation of JNK and p38 requires the activity of upstream MAPK kinases (MAPKKs, e.g., MKK3 and MKK6) and MAPK kinase kinases (MAPKKKs, e.g., apoptosis signal-regulating kinase-1 [ASK-1] and TGF- $\beta$ activated kinase-1 [TAK-1]). Active, phosphorylated forms of JNK and p38 activate transcription factors belonging to the activating protein-1 (AP-1) superfamily (including cJun and activating transcription factor-2 [ATF2]), which drives transcription of proinflammatory genes such as VCAM-1. Proinflammatory MAPK activation has been linked to the development of atherosclerotic lesions, as increased levels of phosphorylated nuclear ATF-2 have been observed in human atherosclerotic plaques [16]. We have also found increased activities of JNK and p38 at atherosusceptible sites in murine aorta along with increased expression of VCAM-1, indicative of a proinflammatory phenotype [14].

Proinflammatory signaling also induces MAPK phosphatase-1 (MKP-1), a negative regulator of the MAPK pathway that inactivates p38 and JNK by removing phosphate groups, which may serve to limit the inflammatory response. It is clear that shear stress is an important factor in determining the expression pattern of MKP-1 because exposing cultured cells to unidirectional high shear stress produced a marked induction of MKP-1. We also demonstrated that MKP-1 dampens proinflammatory signaling since silencing MKP-1 function in cultured endothelial cells increased p38 activation and enhanced VCAM-1 expression in response to shear stress. Deletion of MKP-1 using an MKP-1 knockout mouse also revealed increased activity of JNK and p38 and increased expression of VCAM-1 in protected regions of the mouse aorta. Our findings present a novel molecular mechanism contributing to the spatial distribution of vascular inflammation and atherosclerosis, where high shear stress protects arteries from inflammation by inducing persistent endothelial expression of MKP-1, which suppresses the activities of p38 and JNK [14].

Other reports have identified several other intermediaries in the JNK/p38 MAPK pathway that are negatively regulated by shear stress and that may also serve to dampen proinflammatory signaling in regions of high shear stress. In cultured cells, for example, exposure to high laminar shear stress reduces the activation of ASK-1 (a MAPKKK) by promoting association with its inhibitory binding protein, depicted in Figure 3 [17]. High shear stress also reduces JNK activity by blocking cleavage of protein kinase C epsilon (PKC $)$ ] $[18,19]$. In addition, the influence of shear stress on JNK activation may be influenced by the nature of the subendothelial matrix [20], underlying the complex interplay of flowregulated and inflammatory signaling pathways.

In subsequent studies we have found that nuclear factor erythroid 2-related factor (Nrf2), an antioxidant (protective) transcription factor, is preferentially activated by high shear stress and may be atheroprotective via negative regulation of the MAPK pathway [21]. In unstimulated cells, Nrf2 is suppressed by binding to kelch-like ECHassociated protein-1 (Keap-1), which targets it for ubiquitination and degradation by the proteasome. Upon stimulation, this interaction is disrupted, leading to dissociation from Keap-1. This results in stabilization and accumulation of $\mathrm{Nrf} 2$ and translocation to the nucleus where Nrf2 can induce the expression of many antioxidant genes (e.g., those encoding heme oxygenase-1, glutathione $S$-transferase, and ferritin).

Given the important role of oxidative mechanisms in vascular inflammation, we recently studied the role of $\mathrm{Nrf} 2$ in regulating endothelial activation. We found that $\mathrm{Nrf2}$ is activated in regions of the mouse aorta exposed to unidirectional high shear stress and protected from atherosclerosis. Only inactive cytoplasmic forms of Nrf2 could be detected in the inner curvature, an area of low/ oscillatory shear stress and susceptible to inflammation and lesion formation. In mice lacking Nrf2, both p38 activity and VCAM-1 expression were significantly increased in the protected region, suggesting that Nrf2 blocks proinflammatory signaling in regions of high shear stress. Parallel in vitro studies confirmed this, where application of unidirectional high shear stress to cultured cells significantly enhanced expression and nuclear activity of $\mathrm{Nrf} 2$ with a simultaneous reduction in VCAM-1 expression. We propose that Nrf2 suppresses 
Figure 3. Inflammatory signaling pathways that are regulated by high shear stress

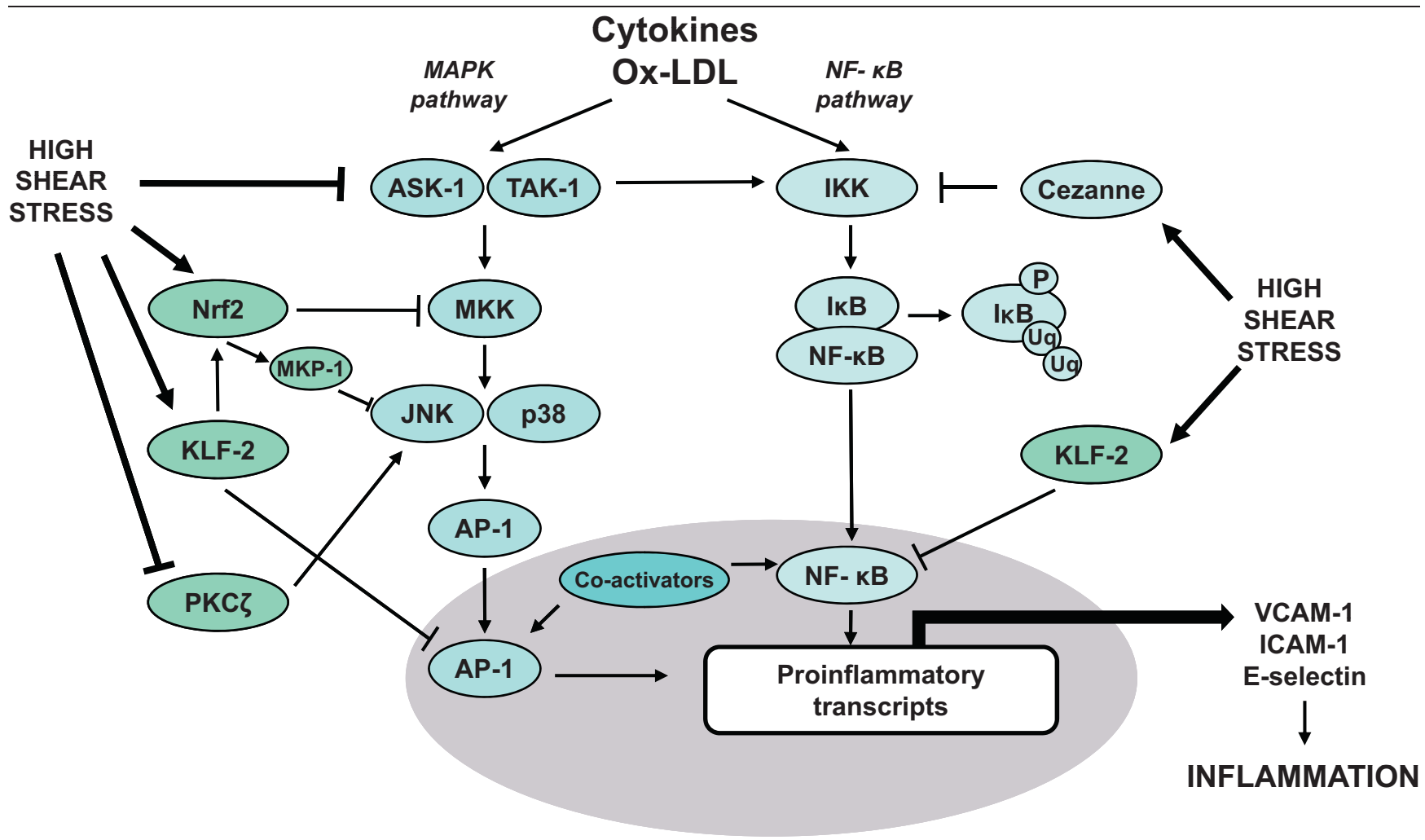

Both the mitogen-activated protein kinase (MAPK) and nuclear factor-kappa-B (NF-kB) signaling pathways are active at sites of the arterial tree exposed to low/oscillatory shear stress and are stimulated by proinflammatory cytokines leading to inflammation and lesion development. At sites of high shear stress, these signaling pathways are inhibited at several levels with a consequent inhibition of inflammation. AP-I, activating Protein-I; ASK-I, apoptosis signal-

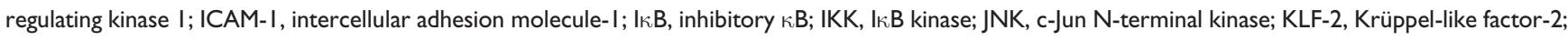
MKP-I, MAPK phosphatase-I; MKK, MAPK kinase; Nrf2, nuclear factor erythroid 2-related factor; Ox-LDL, oxidized low-density lipoprotein; P,

phosphorylation; PKC , protein kinase C epsilon; TAK-I, transforming growth factor (TGF)- $\beta$ activated kinase I; VCAM-I, vascular cell adhesion molecule-I; Uq, ubiquitin.

endothelial activation by targeting the MAPK signaling pathway at two levels: by reducing MKK3/ 6 signaling to p38 and by enhancing the activity of MKP-1. Expression levels of these intermediaries are not altered, rather Nrf2 facilitates increased activity by influencing their redox state; Nrf2 promotes an antioxidant environment that favors the reduced form of MKP-1, which has greater catalytic activity [21]. Thus our studies reveal that Nrf2 is a key determinant of the spatial differences in inflammation and atherosclerosis. Interestingly, genetic deletion of Nrf2 did not completely restore proinflammatory activation at the protected site, suggesting that $\mathrm{Nrf} 2$ works in concert with other anti-inflammatory molecules to inhibit inflammation at sites of high shear stress.

\section{The NF- $\kappa B$ pathway}

The transcription factor NF- $\mathrm{B}$ has been closely linked with cardiovascular health and disease due to its control of multiple processes including immunity, inflammation, cell survival, differentiation and proliferation, and regulation of cellular responses to stress, hypoxia, stretch, and ischemia. NF- $\kappa \mathrm{B}$ signaling in endothelial cells typically leads to the expression of genes that induce recruitment of inflammatory cells to the vessel wall, including ICAM-1 (intercellular adhesion molecule-1), VCAM-1, E-selectin, and cytokines such as TNF- $\alpha$ and IL-1. NF- $K B$ is composed of two subunits, with the p50-p65(RelA) heterodimer most abundant in endothelial cells. In the absence of a stimulus, NF- $\kappa \mathrm{B}$ is bound to an inhibitory molecule, I $\kappa \mathrm{B}$, which masks its nuclear localization sequence and sequesters it in the cytoplasm. NF- $\kappa \mathrm{B}$ is activated by several signaling pathways that cause it to be released from I $\kappa$ B, mostly via activation of an I $\mathrm{k}$ B kinase (IKK), which phosphorylates $\mathrm{I} \kappa \mathrm{B}$, leading to its ubiquitination and subsequent degradation by the proteasome. Once the heterodimer is released, the nuclear localization sequence permits translocation to the nucleus and binding to target sequences on the genome. NF- $\kappa$ B may also itself be 
phosphorylated, which facilitates DNA binding and transcription, but its activity is tightly regulated by negative feedback loops that limit the duration of nuclear localization and hence transcriptional activity. This relies on NF-kB-dependent transcription of $\mathrm{I} \kappa \mathrm{B} \alpha$, which contains a nuclear export sequence, and by binding to NF- $\mathrm{B}$ serves to remove it from the nucleus. Also, activation of the deubiquitinating enzymes A20 and Cezanne by proinflammatory signaling molecules blocks IKK activation and prevents release of NF- $\kappa$ B from I $\kappa B[22,23]$.

Active NF- $\kappa B$ has been identified in atherosclerotic plaques and early lesions along with many NF- $\kappa \mathrm{B}$ regulated molecules, indicating that it is instrumental in atherogenesis. One significant study demonstrated reduced inflammation and plaque formation in atherosclerosis-prone cholesterol-fed mice lacking endothelial expression of IKK $\gamma$, supporting the idea that NF-KB-mediated proinflammatory gene expression plays a causal role in lesion development [24]. Studies in "healthy" mice and pigs revealed that the expression of NF- $\kappa \mathrm{B}$ subunits and regulatory components (p65, I $\kappa B \alpha$, and I $\kappa B \beta$ ) is significantly increased in regions of the aorta exposed to low shear stress compared to high shear stress $[11,12]$. Expression was, however, restricted to the cytoplasm, indicating that NF- $\kappa \mathrm{B}$ was inactive. This suggests that cells exposed to low shear stress are primed for activation upon challenge with a proinflammatory stimulus due to an increased expression of NF- $\kappa B$ subunits [11]. Studies in pigs also showed a reduced expression of negative regulators that switch off NF-kB activity (e.g., Cezanne) at the atherosusceptible site [12]. We have subsequently shown that the expression of Cezanne is shear-sensitive and is increased by unidirectional laminar shear stress [22].

NF- $k B$ transcription factors have the potential to exert dual functions since they can be both antiapoptotic (protective) and proinflammatory. Our own studies, using a parallel plate flow chamber, demonstrated that high laminar shear stress enhanced NF- «B-mediated induction of several antiapoptotic genes, such as BCL2, A20 (TNFAIP3), A1 (BCL2A1), and MnSOD (SOD2), whilst simultaneously dampening proinflammatory activation in response to a proinflammatory stimulus, TNF- $\alpha$ [10]. We therefore conclude that high shear stress can influence the function of NF- $\kappa \mathrm{B}$ by affecting the balance of induction of inflammatory and antiapoptotic genes. Thus endothelial cells at low shear regions are prone to inflammatory activation via multiple mechanisms that influence both the expression and activity of NF- $\kappa B$.

Numerous studies have shown a specific induction of Krüppel-like factor-2 (KLF-2) in response to laminar shear stress and have placed KLF-2 as a key regulator of shear-responsive transcripts and a mediator of the atheroprotective effects of high shear stress $[15,25,26]$. KLF-2 has been shown to attenuate endothelial activation and leukocyte migration in cultured endothelial cells by inhibiting the expression of proinflammatory genes such as those encoding VCAM-1 and E-selectin. The suppressive effects of KLF-2 on inflammatory activation were attributed to its ability to sequester critical coactivators of NF- $\kappa \mathrm{B}$, cyclic AMP response element-binding protein (CBP) and p300, thereby reducing NF- $\kappa B$ mediated transcription [15].

Anton Horrevoet's group have shown that KLF-2 may also reduce proinflammatory gene expression by inhibiting the MAPK pathway. They demonstrated that KLF-2 inhibits nuclear localization of the ATF-2 transcription factor thereby reducing transcription from AP-1 promoter sites. Similarly, knockdown of KLF-2 with small interfering RNA caused a marked reduction in proinflammatory gene expression in cells without flow [16]. Subsequent studies revealed that KLF-2 inhibits the phosphorylation of JNK, an effect that is dependent on the actin cytoskeleton [27]. Work from this group has also shown that KLF-2 is able to potentiate the atheroprotective effects of flow-induced Nrf2 by enhancing its nuclear translocation and activation [28], indicating that these central regulators of the antiinflammatory effects of shear stress may work in synergy to modulate the transcriptome.

\section{Implications for therapeutic strategies to prevent or treat atherosclerosis}

It is clear that both the MAPK pathway and the NF- $\kappa B$ pathway play a significant role in endothelial activation at atherosusceptible sites and that high shear stress is able to suppress activation of these pathways and protect against lesion development (summarized in Figure 3). KLF-2 and Nrf2 have been identified as central transcriptional regulators of the physiological response to shear stress and are of major interest since pharmacological manipulation could potentially be used to reduce vascular inflammation at atherosusceptible sites and hence slow or prevent lesion formation to reduce morbidity of cardiovascular disease. To this effect, we recently demonstrated that sulforaphane, a naturally occurring organic compound that acts as an antioxidant and is derived in high quantities from broccoli, watercress, and other green vegetables, has atheroprotective actions. We found that sulforaphane, at a relatively low concentration, can reduce inflammatory activation of endothelial cells at atherosusceptible sites by increasing Nrf2 activity via inhibition of Keap-1 [21]. Thus sulforaphane is an example of a targeted therapeutic 
intervention that enhances expression of protective, antiinflammatory transcripts at atherosusceptible sites and may, in the future, have clinical utility for the prevention or treatment of cardiovascular inflammation. Since sulforaphane can be found in abundance in many green vegetables, this finding is consistent with the concept that eating broccoli and other green vegetables can lower the risk of atherosclerosis.

Several groups have recently studied the effects of statins (cholesterol-lowering drugs) on endothelial physiology and observed that statins, independently of their lipidlowering actions, can suppress numerous inflammatory genes via the induction of KLF-2 [29,30]. Interestingly, exposure of endothelial cells to laminar shear stress enhanced the responsiveness of endothelial cells to statins, whereas exposure to disturbed blood flow had the opposite effect [31]. These data highlight the influence of mechanical forces on vascular responses to pharmacological agents and suggest that statins may preferentially influence high-shear regions of the arterial tree.

\section{Conclusions}

Genome-wide approaches are now required to further define the molecular mechanisms that control the spatial distribution of vascular inflammation and atherogenesis. Analysis of the endothelial transcriptome at sites of low/oscillatory and high shear stress will reveal specific transcripts that are differentially regulated in response to shear stress and could, in time, be targeted for pharmacological manipulation. This approach will also shed light on the mechanisms by which shear stress modulates endothelial apoptosis, proliferation, and thrombogenicity, all of which contribute to the pathogenesis of atherosclerosis. Better understanding of these mechanisms will enable targeted therapeutic interventions that promote a protective phenotype in atherosusceptible regions in order to slow or even halt the progression of cardiovascular disease.

\section{Abbreviations}

AP-1, activating protein-1; ASK-1, apoptosis signalregulating kinase-1; ATF-2, activating transcription factor-2; CBP, cyclic AMP response element-binding protein; ECM, extracellular matrix; ERK, extracellular signal-regulated kinase; E-selectin, endothelial selectin; ICAM-1, intercellular adhesion molecule-1; I $\kappa B$, inhibitory $\kappa \mathrm{B}$; IKK, I $\kappa \mathrm{B}$ kinase; IL, interleukin; JNK, c-Jun Nterminal kinase; Keap-1, kelch-like ECH-associated protein-1; KLF-2, Krüppel-like factor-2; LDL, low-density lipoprotein; MAPK, mitogen-activated protein kinase; MAPKK, MAPK kinase; MAPKKK, MAPK kinase kinase; MKP-1, MAPK phosphatase-1; MnSOD, manganese superoxide dismutase; NF- $\kappa \mathrm{B}$, nuclear factor-kappa-B;
Nrf2, nuclear factor erythroid 2-related factor; PECAM-1, platelet endothelial cell adhesion molecule-1; $\mathrm{PKC \zeta}$, protein kinase $\mathrm{C}$ epsilon; TAK-1, transforming growth factor (TGF)- $\beta$ activated kinase- 1 ; TNF- $\alpha$, tumor necrosis factor-alpha; VCAM-1, vascular cell adhesion molecule-1; VE-cadherin, vascular-endothelial cadherin; VEGFR-2, vascular endothelial growth factor receptor- 2 .

\section{Competing interests}

The authors declare that they have no competing interests.

\section{Acknowledgements}

Our studies were funded by the British Heart Foundation.

\section{References}

I. Adiguzel E, Ahmad PJ, Franco C, Bendeck MP: Collagens in the progression and complications of atherosclerosis. Vasc Med 2009, 14:73-89.

2. Tricot O, Mallat Z, Heymes C, Belmin J, Lesèche G, Tedgui A: Relation between endothelial cell apoptosis and blood flow direction in human atherosclerotic plaques. Circulation 2000, I 0 |:2450-3.

3. Caro CG, Fitz-Gerald JM, Schroter RC: Arterial wall shear and distribution of early atheroma in man. Nature 1969, 223: $1159-60$.

4. Weinberg PD: Rate limiting steps in the development of atherosclerosis; the response-to-influx theory. J Vasc Res 2004, 4I:I-I7.

5. Malek AM, Alper SL, Izumo S: Hemodynamic shear stress and its role in atherosclerosis. JAMA 1999, 282:2035-42.

6. Tzima E, Irani-Tehrani M, Kiosses WB, Dejana E, Schultz DA, Engelhardt B, Cao G, DeLisser H, Schwartz MA: A mechanosensory complex that mediates the endothelial response to fluid shear stress. Nature 2005, 437:426-31.

FI000 Factor 15

Evaluated by Dietmar Vestweber 27 Sep 2005, Christopher Marshall 04 Oct 2005, Mark Ginsberg 24 Nov 2005

7. Tzima E, Angel del Pozo M, Shattil SJ, Chien S, Schwartz MA: Activation of integrins in endothelial cells by fluid shear stress mediates Rho-dependent cytoskeletal alignment. EMBO J 200I, 20:4639-47.

8. Civelek M, Manduchi E, Riley RJ, Stoeckert CJ, Davies PF: Chronic endoplasmic reticulum stress activates unfolded protein response in arterial endothelium in regions of susceptibility to atherosclerosis. Circ Res 2009, 105:453-6I.

FI000 Factor 6

Evaluated by Dorian Haskard 15 Oct 2009

9. Sheikh S, Rainger GE, Gale Z, Rahman M, Nash GB: Exposure to fluid shear stress modulates the ability of endothelial cells to recruit neutrophils in response to TNF- $\alpha$ : $A$ basis for local variations in vascular sensitivity to inflammation. Blood 2003, 1 02:2828-34.

10. Partridge J, Carlsen H, Enesa K, Chaudhury H, Zakkar M, Luong LA, Kinderlerer A, Johns M, Blomhoff R, Mason JC, Haskard DO, Evans PC: Laminar shear stress acts as a switch to regulate divergent functions of NF- $\kappa$ B in endothelial cells. FASEB J 2007, 21:3553-61.

II. Hajra L, Evans Al, Chen M, Hyduk SJ, Collins T, Cybulsky MI: The NF- $\kappa$ B signal transduction pathway in aortic endothelial cells is primed for activation in regions pre-disposed to atherosclerotic lesion formation. PNAS 2000, 97:9052-7. 
12. Passerini AG, Polacek DC, Shi C, Francesco NM, Manduchi E, Grant GR, Pritchard WF, Powell S, Chang GY, Stoeckert CJ Jr, Davies PF: Coexisting proinflammatory and antioxidative endothelial transcription profiles in a disturbed flow region of the adult porcine aorta. Proc Natl Acad Sci U S A 2004, I0I:2482-7.

13. Jongstra-Bilen J, Haidari M, Zhu S-N, Chen M, Guha D, Cybulsky MI: Low-grade chronic inflammation in regions of the normal mouse arterial intima predisposed to atherosclerosis. J Exp Med 2006, 203:2073-83.

FI000 Factor 8

Evaluated by Klaus Ley 15 Aug 2006

14. Zakkar M, Chaudhury H, Sandvik G, Enesa K, Luong LA, Cuhlmann S, Mason JC, Krams R, Clark AR, Haskard DO, Evans PC: Increased endothelial MKP-I expression suppresses proinflammatory activation at sites that are resistant to atherosclerosis. Circ Res 2008, 103:726-32.

15. SenBanerjee S, Lin Z, Atkins GB, Greif DM, Rao RM, Kumar A, Feinberg MW, Chen Z, Simon DI, Luscinskas FW, Michel TM, Gimbrone MA Jr, García-Cardeña G, Jain MK: KLF2 is a novel transcriptional regulator of endothelial proinflammatory activation. J Exp Med 2004, 199:1305-15.

16. Fledderus JO, van Thienen JV, Boon RA, Dekker RJ, Rohlena J, Volger OL, Bijnens AP, Daemen MJ, Kuiper J, van Berkel TJ, Pannekoek H, Horrevoets A]: Prolonged shear stress and KLF2 suppress constitutive proinflammatory transcription through inhibition of ATF2. Blood 2007, 109:4249-57.

17. Liu Y, Yin G, Surapisitchat J, Berk BC, Min W: Laminar flow inhibits TNF-induced ASKI activation by preventing dissociation of ASKI from its inhibitor 14-3-3. J Clin Invest 200I, 107:917-23.

18. Magid R, Davies PF: Endothelial protein kinase c isoform identity and differential activity of $P K C \zeta$ in an atherosusceptible region of porcine aorta. Circ Res 2005, 97:443-9.

19. Garin G, Abe J, Mohan A, Lu W, Yan C, Newby AC, Rhaman A, Berk BC: Flow antagonizes TNF- $\alpha$ signaling in endothelial cells by inhibiting caspase-dependent PKC $\zeta$ processing. Circ Res 2007, 101:97-105.

20. Hahn C, Orr AW, Sanders JM, Jhaveri KA, Schwartz MA: The subendothelial extracellular matrix modulates JNK activation by flow. Circ Res 2009, 104:995-1003.

21. Zakkar M, van der Heiden K, Luong LA, Chaudhury H, Cuhlmann S, Hamdulay SS, Krams R, Edirisinghe I, Rahman I, Carlsen H, Haskard DO, Mason JC, Evans PC: Activation of Nrf2 in endothelial cells protects arteries from exhibiting a proinflammatory state. Arterioscler Thromb Vasc Biol 2009, 29:185I-7.

22. Enesa K, Zakkar M, Chaudhury H, Luong LA, Rawlinson L, Mason JC, Haskard DO, Dean JL, Evans PC: NF-kappaB suppression by the deubiquitinating enzyme Cezanne: a novel negative feedback loop in proinflammatory signaling. J Biol Chem 2008, 283:7036-45.

23. Harhaj EW, Dixit VM: Deubiquitinases in the regulation of NF-KB signaling. Cell Res 20II, 2I:22-39.

24. Gareus R, Kotsaki E, Xanthoulea S, van der Made I, Gijbels MJ, Kardakaris R, Polykratis A, Kollias G, de Winther MP, Pasparakis M: Endothelial cell-specific NF- $\kappa$ B inhibition protects mice from atherosclerosis. Cell Metabolism 2008, 8:372-83.

25. Parmar KM, Larman HB, Dai G, Zhang Y, Wang ET, Moorthy SN, Kratz JR, Jain MK, Gimbrone MA Jr, García-Cardeña G: Intergration of flow-dependent endothelial phenotypes by Kruppel-like factor 2. J Clin Invest 2006, I 16:49-58.

26. Wang N, Miao H, Li YS, Zhang P, Haga JH, Hu Y, Young A, Yuan S, Nguyen P, Wu CC, Chien S: Shear stress regulation of Kruppellike factor 2 expression is flow-pattern specific. Biochem Biophys Res Comm 2006, 341:|244-5|.

27. Boon RA, Leyen TA, Fontin RD, Fledderus JO, Baggen JM, Volger OL, van Nieuw Amerongen GP, Horrevoets AJ: KLF2-induced actin shear fibers control both alignment to flow and JNK signaling in vascular endothelium. Blood 2010, I 1 5:2533-42.

28. Fledderus JO, Boon RA, Volger OL, Hurtilla $H$, Ylä-Herttuala $S$, Pannekoek H, Levonen AL, Horrevoets AJG: KLF2 primes the antioxidant transcription factor Nrf2 for activation in endothelial cells. Arterioscler Thromb Vasc Biol 2008, 28:1339-46.

29. Parmar KM, Nambudiri V, Dai G, Larman HB, Gimbrone MA Jr, García-Cardeña G: Statins exert endothelial atheroprotective effects via the KLF2 transcription factor. J Biol Chem 2005, 280:267|4-9.

30. SenBanerjee S, Mir S, Lin Z, Hamik A, Atkins GB, Das H, Banerjee P, Kumar A, Jain MK: Kruppel-like factor 2 as a novel mediator of statin effects in endothelial cells. Circulation 2005, I I 2:720-6.

3I. Ali F, Zakkar M, Karu K, Lidington EA, Hamdulay SS, Boyle J], Zloh M, Bauer A, Haskard DO, Evans PC, Mason JC: Induction of the cytoprotective enzyme heme oxygenase-I by statins is enhanced in vascular endothelium exposed to laminar shear stress and impaired by disturbed flow. J Biol Chem 2009, 284: $18882-92$ 